\title{
Guiding Ethics Review in Pharmacy Education Research and Scholarship at UBC: Clarifying the Unclear
}

Simon P. Albon, PhD and Franklin Hu, PharmD Candidate

University of British Columbia, Faculty of Pharmaceutical Sciences, Vancouver, British Columbia

\begin{abstract}
While it goes without saying that ethically sound practices are imperative for high-quality educational scholarship, institutional ethics guidance is often unclear about how to treat educational scholarship generally, and quality improvement/assurance studies and the scholarship of teaching and learning, specifically. Amongst health profession education researchers, including those in pharmacy, this lack of clarity has led to confusion regarding existing ethics governance and ambivalence regarding ethics requirements. Drawing on the experiences of one pharmacy school in western Canada, this commentary describes an ethics vetting guide developed explicitly to address current uncertainty about ethics requirements for pharmacy education scholarship. Clarifying the problem, describing the guide, and exploring what was learned along the way provide a basis for re-centering ethics in the development of scholarly projects and decision-making regarding formal ethics review. The importance of instilling ethical intelligence, delineating research from quality improvement/assurance work, and addressing current gaps in ethics oversight and governance of educational scholarship are among key lessons learned during guide development along with suggestions for new institutional ethics guidance directly targeting educational scholarship to supplement current national guidelines.
\end{abstract}

Keywords: ethics review, ethics vetting guide, educational scholarship, quality assurance/improvement, scholarship of teaching and learning

\section{The Issue}

You might be asking: why would we want to bring up the subject of research ethics, let alone write a paper about it? Doesn't it go without saying that formal ethics review is imperative in all research studies involving human subjects, including our students? In the wake of the atrocities of World War II, the Nuremberg Code (1947) and the Declaration of Helsinki (1964) laid the groundwork for seminal research ethics documents such as the Belmont Report in the US (1979) and Canada's Tri-Council Policy Statement: Ethical Conduct for Research Involving Humans (TCPS; 1998)[1][2]. Adhering to internationally-accepted models of ethics governance involving multidisciplinary, independent research ethics boards (REBs), these foundational documents provide rigorous guidelines for ensuring respect for human dignity in all research activities and in particular, for biomedical and behavioral studies. Within local jurisdictions, REBs are tasked with navigating the complex landscape of research involving humans with stringent oversight and levels of scrutiny that balance necessary human protections against societal benefits. For those more interested in educational scholarship and the Scholarship of Teaching and Learning (SoTL), the TCPS, now in its third iteration, ${ }^{1}$ also includes guidance on exemptions, such as research using publicly available data and quality assurance and improvement studies in educational settings. Article 2.5 of the Tri-Council Policy Statement 2 (TCPS 2), for example, states that "Quality

Corresponding author: Simon P. Albon, PhD

Professor of Teaching

University of British Columbia

Faculty of Pharmaceutical Sciences

Phone: 604-822-2497; Email: simon.albon@ubc.ca assurance and quality improvement (QA/QI) studies, program evaluation activities, and performance reviews, or testing within normal educational requirements when used exclusively for assessment, management or improvement purposes, do not constitute research for the purposes of this Policy, and do not fall within the scope of REB review," (p. 18). In addition, Article 2.5 includes a blanket safeguard common for biomedical and behavioral research, "When in doubt about the applicability of the article to their studies, researchers should consult their REBs," (p. 18).

On the surface, Article 2.5 may seem clear, particularly for SoTL scholars deeply engaged in advancing the quality of educational practices and programs through $\mathrm{QA} / \mathrm{Q}$ I efforts. In reality however, interpretation of this article on research-intensive university campuses, like the University of British Columbia (UBC), has created a great deal of confusion and uncertainty. ${ }^{2}$ While there is no dispute about the need to follow the ethical principles and practices laid out in established TCPS 2 and REB governance, lack of clarity about what constitutes research and QA/QI work, what scope of classroom and program-based SoTL research requires formal ethics review, and whether or not ethics-exempt studies could be published led to many unfortunate consequences.

In the author's Faculty, for example, the default positions of many pharmacy education researchers include: undertaking projects without ethics review or inquiring about ethics requirements retrospectively, completing scholarly projects without dissemination or publication, not completing projects at all, particularly when faced with the extensive ethics application process, or submitting full ethics applications for every project to hedge their bets. At a time when pedagogical 
research is gaining traction and legitimacy in our Faculty and more broadly across campus (and in academic pharmacy in Canada), this lack of clarity presents a barrier to advancement of program quality and career growth amongst pharmacy education researchers. In the absence of clear guidelines from UBC's Office of Research Ethics and our local REB, ethics requirements for $\mathrm{QA} / \mathrm{Q}$ I work and SoTL research remains murky and contested. There is also a general ambivalence about ethics requirements.

Perhaps not surprisingly, the experiences within our Faculty and on the UBC campus are not new. The conversation about ethics requirements for $\mathrm{QA} / \mathrm{QI}$ and SoTL research has been festering for years. Within the medical education community for example, Kevin Eva, ${ }^{3}$ editor of Medical Education captures this ethos by asking "Is there any question that raises a broader array of emotions than 'Did you secure ethics approval prior to starting your research project'” (p. 194) while Pugsley and Dornan ${ }^{4}$ lament the often onerous ethics review requirements for pedagogical research as "using a sledgehammer to crack a nut" (p. 726). ten Cate ${ }^{5}$ and McLachlan and McHarg ${ }^{6}$ bookend a spectrum of arguments questioning the ethical differences between medical education and medical research, and the need for full ethics review for routinely collected data. Frameworks for the ethics review of health professions education research have been proposed $4,7,8$ that may address the negative impacts of unwieldy and inflexible research oversight and governance, but provide little concrete practical guidance. ${ }^{9}$ Within the pharmacy education research landscape, McLaughlin and colleagues ${ }^{10}$ emphasize that meaningful educational research within colleges and schools of pharmacy must include ethical considerations, but suggest that the need for full ethics review for all pharmacy education research may be context specific. In higher education contexts generally, Dalhousie University's REB has recently met this challenge head-on convening an interdisciplinary working team to create specific REB protocols and governance inclusive of ethically sound SoTL research practices and policies. ${ }^{2}$ To date, our Faculty and university have been silent on this issue.

To fill this gap, we partnered with UBC's Office of Research Ethics (ORE) and Centre for Teaching Learning and Technology (CTLT) as well as pharmacy education researchers to create an ethics vetting guide to help clarify ethics review decisionmaking within our Faculty and the broader university community. The intent of this paper is to describe the product we created and the lessons learned along the way, with the hope of equipping faculty with the knowledge and tools necessary to engage in SoTL.

\section{What We Created}

The ethics vetting guide was created in the fall of 2019. Aligned with good scholarly practices, ${ }^{11}$ guide development required comprehensive knowledge of the nature and intricacies of UBC's Behavioral Research Ethics Board (BREB) review processes and its relation to educational research, QA/QI, and
SoTL studies. To build sufficient knowledge, we: 1) completed Canada's TCPS 2 tutorial $^{12}$ on research ethics involving humans and reviewed UBC's formal BREB application process; 2) conducted a review of the health professions and higher education literature for information and processes related to ethics review of educational scholarship, QA/QI and SoTL research [3]; 3) researched and collected existing ethics documents created by the UBC community to address the confusion and uncertainty surrounding ethics review of education-related scholarship, and; 4) engaged with UBC's ORE, CTLT, and pharmacy education researchers for iterative feedback and guide development.

The TCPS 2 tutorial is an interactive eight-module, online course that establishes foundational ethical principles and practices for research involving humans. Although focused primarily on medical research, as mentioned previously, some ethics exemptions are also discussed. The MK-Ultra, Tuskagee Syphilis and Stanford Prison studies are among several Canadian and US historical events used to highlight the critical importance of ethically-sound research governance and oversight today. At UBC, one-time completion of the TCPS 2 tutorial is mandatory for any human subject-related inquiry with submission of the completion certificate a requirement for all REB reviews. The BREB application comprises a 12-section detailing of study investigators, timelines, recruitment, procedures, consent processes, risks/benefits and data security among other key design features; the ORE considers the BREB application a proxy for how the research will be conducted and the degree to which established ethical standards will be met.

The literature review focused on health professions and higher education research published in the last decade addressing issues and barriers constraining or complicating ethics governance of educational scholarship and related ethics vetting processes. Health professions articles came primarily from the medical education literature in the form of commentaries and recommendations; no operationalized ethics vetting processes for education-related projects were described. A small body of research on ethically sound SoTL research in higher education provided additional insight. ${ }^{2}$ Two UBC-specific tools were identified and aimed at helping educators decide whether BREB review was warranted: a checklist by ORE ${ }^{13}$ and a CTLT decision-making process. ${ }^{14}$ While the scholarly literature and the UBC tools provided a welcomed, high-level framing of important background and issues, together they provided little clarity on the nuances of ethics decision-making related to educational scholarship. For example, defining "research" in the educational scholarship space, based on the TCPS 2 definitions, was surprisingly challenging. Consequently, we partnered with UBC's ORE, CTLT, and pharmacy educators in multiple cycles of critique and feedback to develop a coherent and holistic ethics vetting guide that clarified the complexities of BREB review in pedagogical studies including definitions of research. Consensus amongst collaborators was very positive indicating that the guide filled a 
resource gap regarding ethics decision-making on the UBC campus and in our Faculty.

The guide[4] itself consists of four sections: I) a narrative providing essential background information and definitions; II) a short section on standards of ethical practice for educational scholarship; III) a stepwise ethics decision-making process, and; IV) an appendix providing a review of key principles required for ethical conduct of pedagogical research and examples showing how the guide can be applied. Table 1 provides an overview of each section while the complete guide can be found at the UBC Office of Research Ethics Website (see "Ethics Considerations for Educational Scholarship"). The guide is considered a living document with additions and revisions anticipated as projects develop and our understanding of ethics governance of educational scholarship evolves.

Table 1. Overview of the ethics vetting guide

Section

Description

I Situates the importance of educational scholarship in the Faculty's research mandate and the associated historical challenges of formal BREB review decision-making at UBC. Significant effort is dedicated to clarifying key confounders in decision-making related to the TCPS 2 classifications of research and QA/QI work. Aspects of study design, such as locus of attention, data sources, expectations of faculty and students, theoretical frameworks, generalizability, and dissemination priorities, provide additional criteria for delineation.

II Reminds pharmacy education researchers about the importance of ethical conduct of research involving humans regardless of project classification. An associated appendix provides accepted standards of ethical practice for all education-related studies involving humans. Principles described include consent, informing students, confidentiality and anonymity, vulnerability, and beneficence.

III Describes a four-step process that engages pharmacy education researchers in thinking critically about ethically-sound research, and implications for their studies. In addition to getting started activities, a questionnaire helps classify projects as research or QA/QI studies according to TCPS 2 definitions and make decisions about whether or not formal ethics review is required. Further consultation and a focus on dissemination practices completes the process.

IV Provides the supporting appendix. Along with the key principles of ethical research conduct mentioned in Section II, examples of how the guide has been applied are included. Research projects requiring formal BREB review and ethicsexempt QA/QI studies completed by pharmacy education researchers in our Faculty are highlighted. Entries include project titles and abstracts, project team members, questionnaire answers with final decisions, and specific language acknowledging REB decisions (regarding approvals and exemptions) for dissemination purposes in presentations, journal article submission requirements, and publications.

Figure 1. The four-step ethics decision-making process.

Step 1. Complete

the TCPS 2 tutorial

and RISe profiles for

all team members

including students.
Step 2. Complete

the ethics

questionnaire.

Establish an initial

decision regarding

the need for formal

ethics review.
Step 3. Seek peer

review from an

experienced

educational

researcher. Confirm

the decision

regarding formal

ethics review.
Step 4. Provide appropriate

acknowledgements

and language

regarding ethics

decisions for

dissemination

purposes. 
With Sections I and II providing important context for pharmacy education researchers, Section III is the focal point of the guide, describing the four-step ethics decision-making process. As illustrated in Figure 1, Step 1 introduces pharmacy education researchers to research ethics through mandatory completion of the TCPS 2 tutorial and creation of a UBC Research Information System (RISe) account.[2] The intent is to center ethics as imperative for quality scholarship along with the institutional expectations regarding the ethical conduct of all research at the university.

Step 2 focuses on decisions about project classification (ie, research versus $Q A / Q$ I work) and formal ethics review. To help clarify, pharmacy education researchers are asked to develop defensible answers to seven questions related to the ethical conduct of their research ideas and projects (Table 2). The questions were developed based on recommendations from the research literature ${ }^{3-8}$ and the experiences of the UBC community. ${ }^{13-14}$ While most questions require "Yes/No" answers, researchers are encouraged to develop rationales for their answers based on careful consideration of ethical implications of their research questions and study designs. Completing the questionnaire ends in a decision point regarding the need for formal ethics review (see Table 2 key).

Table 2. Ethics classification questionnaire

\section{$\begin{array}{ll}\text { Questions }^{*} & \text { Answers }\end{array}$}

1. Does the project/study fit the TCPS' definition of "research" or "quality assurance/improvement"? Explain.

2. Will any of the concepts of consent, informing students, confidentiality, vulnerability, and beneficence be compromised at any time during the project?

3. Is there uncertainty of possible risk to any participant through experiencing either physical or psychological distress or discomfort?

4. Is the project design and methodology rigorous enough to statistically support generalizations beyond the particular context and/or population that will participate in the project?

5. Is the project funded by (or being submitted to) a grant/award competition from a funding agency that requires research ethics review?

6. Does the project involve "randomization" to contrast interventions to participants or other systematic sampling techniques to divide participants into different groups?

7. Does the project involve a comparison of interventions or processes and "control" settings or groups either to test a new intervention or to assess the effectiveness of a process change?

* Decision point: If you are clear about your answer to question 1 and answered "No" to questions 2-7 then your project is likely a quality assurance/improvement project/study and may not require formal BREB review. If you are unsure about your answer to question 1 and/or answered "Yes" to any of questions 2-7 then your project may require a formal BREB application and review.

Communicating decisions to others is the focus of Step 3. In particular, researchers are encouraged to discuss their studies and decisions with experienced colleagues for confirmation and clarifying uncertainties. This step may also involve contacting ORE for further input and support for development of formal ethics applications, if needed. The last stage of the decisionmaking process, Step 4, emphasizes dissemination, in particular, the appropriate ethics acknowledgements and requirements for presentations and journal submissions.[5]

\section{What We Learned}

Development of the ethics vetting guide was more difficult than expected. While the initial goal of addressing long standing uncertainty about formal ethics review seemed straight forward, writing the guide proved challenging. Clarifying definitions, creating the classification questionnaire, assembling the appendix and writing a narrative that reflected the critical importance of ethically-sound scholarship required unexpected rounds of feedback and editing, over months not weeks. Imperative was presenting ethics review not as BREB bureaucracy, but as integral to the planning and conduct of all 
scholarly initiatives and vital for the professional growth of educators and educational scholars. ${ }^{4,9}$ Instilling a culture of ethical intelligence ${ }^{15}$ that is, the active process of informed and continuous awareness about the ethical challenges and researcher responsibility towards study participants, was crucial. At a time when interest and engagement in educationrelated scholarship was expanding rapidly in both our Faculty and across campus, reinforcing the central principles and practices of ethical conduct in educational inquiry, how to treat $Q A / Q$ I work specifically, and confronting ambivalence about BREB requirements was timely for enabling both high-quality scholarship and a strong research community. For educators interested in advancing the quality of their practices and programs through scholarship, the guide fills a resource gap that encourages systematic review of ethical considerations during study development, while providing strategic decisionmaking about ethical oversight requirements. Meant to be user friendly and practical, the guide clarifies the unclear elements of TCPS 2 guidance, safeguards appropriate levels of ethical scrutiny, and applies to a broad range of educational initiatives.

Delineating research from $Q A / Q$ I work was by far, the most contentious aspect of guide development. Many intense conversations with ORE personnel, CTLT staff, and pharmacy educators and researchers were required for clarity to emerge. The problem appears twofold. The first is that the line between research and $Q A / Q I$, as defined by the TCPS 2, has become increasingly blurred in health professions settings. ${ }^{16}$ Today, $\mathrm{QA} / \mathrm{Q}$ and research studies share common language, protocols, data analysis techniques, and purposes. The credibility of QA/QI study findings, like research, are dependent on systematic investigation and methodological rigour. In terms of purposes, generating new knowledge and generalizability, once defining distinctions between the two, have also been questioned. ${ }^{16}$ Writing for an audience of health education researchers, Regehr ${ }^{17}$ has argued eloquently that generating contextspecific new knowledge is imperative for improving contemporary health professions education and that understanding learning context complexity outweighs the need for generalizable findings. From this perspective, systematic and rigorous QA/QI work in health professions education, even for routinely collected data, is vital for understanding program complexity well. Interestingly, the TCPS 2 definition of research has removed the previous requirement for generalizability in recognition that new knowledge discoveries are often contextspecific. ${ }^{1}$ Automatically relegating QA/QI studies as ethicsexempt may need careful reconsideration in the future.

The second reason for contention appears to be one of optics; that is, pharmacy education researchers do not see themselves or their scholarship represented in the TCPS 2 guidelines. In addition to the problematic delineations between research and QA/QI described above, SoTL is not mentioned at all and instead, is subsumed within generic language related to the dual role of educators in classroom research and the potential risks to students of undue influence.[6] For pharmacy education researchers focused on QA/QI and SoTL work, an important question has emerged, "What do we get to call research?" This issue and its negative consequences have not gone unnoticed, particularly in higher education contexts. Stockley and Balkwill, ${ }^{18}$ for example, insist that the absence of any acknowledgement or concrete guidance regarding educational scholarship and SoTL in the TCPS 2 may be driving ambivalence and aversion towards ethics requirements amongst educators. MacLean and Poole' ${ }^{19}$ seminal work on the teacher-researcher role in SoTL suggests the TCPS 2 leaves tensions between classroom research and teaching unrecognized or unexplored to the detriment of student fairness, safety, and quality scholarship. Particularly important is unacknowledged power differentials between the teacherresearcher and student-participants that can coerce students to participate for the sake of their grades and undermine the integrity and validity of the study findings. ${ }^{19}$ Like Poole, ${ }^{20}$ we would argue that seeing the scholarly endeavors of pharmacy education researchers reflected in national and university ethics guidelines not only signals the value of this work to institutions and society, but is critical for its ongoing development, quality and legitimacy. Addressing this issue directly, the guide answers the question for pharmacy education researchers by encouraging them to consider all their educational scholarship as research, while paying special attention to categorization according to TCPS 2 definitions for dissemination purposes and ethically-sound research practices.

The final take-away from developing the ethics vetting guide can be summarized in the following questions, why has it taken so long to recognize the obvious? Or have we become so resigned to current ethics review processes that they go unquestioned? For these authors, creating the guide has disrupted our complacency about ethics review of educational scholarship and broadened our thinking about gaps in available guidance. Although a required starting point in Canada, the TCPS 2 was not originally developed to guide ethically sound educational scholarship. As Schnurr and Taylor ${ }^{2}$ claim regarding the TCPS 2's biomedical focus, "SoTL represents an ethically fuzzy arena of scholarship" (p.2). In higher education contexts, research over the past decade has clarified important ethical issues underlying the student-instructor relationship in SoTL, such as power dynamics, consent, fairness and equity, and confidentiality 19,21 and led to university-endorsed guidance documents, such as those developed by the University of Calgary. Currently, no equivalent guidance has been reported in the pharmacy education literature or developed at UBC. In addition, available ethics guidance for SoTL work addresses only one of five diverse domains of SoTL research (the didactic domain involving students) ${ }^{22}$ and does not include ethical considerations for $Q A / Q I$ work specifically. We would argue that instead of trying to fit educational scholarship into TCPS 2 definitions and guidelines, new, innovative, complementary policies and protocols aimed explicitly at ethics governance and oversight for educational research, QA/QI and SoTL studies are needed. As a call to action, our next task will be to work with 
UBC's ORE to develop specific guidance that addresses these issues while speaking directly to pharmacy education researchers and others across campus to ensure continued growth of quality scholarship and academic programs, a strong research community, and engagement of students as partners.

\section{Final Thoughts}

Based on what we have done and what is left to do, writing the ethics vetting guide is not over. Our well-intentioned process of clarifying the unclear about ethics requirements for educational scholarship has opened up unexpected gaps needing to be filled. As a living document, the ethics vetting guide promises to shift and evolve as our understanding of ethics governance of educational scholarship grows and changes. In the short term, the guide addresses long-standing concerns regarding ethics oversight while reinforcing the importance of ethically sound principles, practices, and decision-making required for quality scholarship. In the long term, the guide and subsequent iterations will help build capacity and legitimacy of educational scholarship in our Faculty, university, and academic pharmacy in Canada while enhancing the scholarship of pharmacy education researchers for the betterment of students, faculty, programs and patients.

Acknowledgements: The authors would like to acknowledge the support and encouragement of Jean Ruiz and Maria Valente of UBC's Office of Research Ethics, Dr. Kari Grain of UBC's Centre for Teaching, Learning and Technology, and colleagues in the UBC Faculty of Pharmaceutical Sciences for their on-going commitment to developing the ethics vetting guide. The authors also thank Dr. Jacqui McLaughlin at the UNC Eshelman School of Pharmacy, and Dr. Andrea Webb of UBC's Faculty of Education for valuable editorial suggestions on the commentary.

\section{Conflicts of Interest: None}

Funding/support: None

\section{References}

1. Canadian Institutes of Health Research, Natural Sciences and Engineering Research Council of Canada, and Social Sciences and Humanities Research Council. Tri-Council Policy Statement: Ethical Conduct for Research Involving Humans. Ottawa, ON: Interagency Secretariat on Research Ethics; 2018:1-203. https://ethics.gc.ca/eng/documents/tcps2-2018-eninteractive-final.pdf. Accessed August 22, 2021.

2. Schurr MA, Taylor A. Bridging the Gap between the Research Ethics Board and the Scholarship of Teaching and Learning. CJSoTL. 2019;10(1): Article 12. doi.org: 10.5206/cjsotl-rcacea.2019.1.8003

3. Eva KW. Research ethics requirements for Medical Education. Med Educ. 2009;43(3):194-5. doi: 10.1111/j.1365-2923.2008.03285.x
4. Pusley L, Dornan T. Using a sledgehammer to crack a nut: clinical ethics review and medical education research projects. Med Educ. 2007;41(8):726-728. doi: 10.1111/j.1365-2923.2007.02805.x.

5. ten Cate $\mathrm{O}$. Why the ethics of medical education research differs from that of medical research. Med Educ. 2009;43(7):608-610. doi: 10.1111/j.13652923.2009.03385.x.

6. McLachlan JC, McHarg J. Ethical permission for the publication of routinely collected data. Med Educ. 2005;39(9):944-948. doi: 10.1111/j.13652929.2005.02223.x.

7. Eikelboom JI, ten Cate OT, Jaarsma D, Raat JA, Schuwirth $\mathrm{L}$, van Delden JJ. A framework for the ethics review of education research. Med Educ. 2012;46(8):731-733. doi: 10.1111/j.1365-2923.2012.04293.x

8. Bradbury-Jones C, Alcock J. Nursing students as research participants: a framework for ethical practice. Nurse Educ. Today. 2010;30(2):192-196. doi:10.1016/j.nedt.2009.07.013

9. Robinson L, Drewery S, Ellershaw J, Smith J, Whittle S, Murdoch-Eaton D. Research governance: impeding both research and teaching? A survey of impact on undergraduate research opportunities. Med Educ. 2007;41(8):729-736. doi: 10.1111/j.13652923.2007.02776.x

10. McLaughlin JE, Dean MJ, Mumper RJ, Blouin RA, Roth MT. A roadmap for educational research in pharmacy. Am J Pharm Educ. 2013;77(10):218. doi: 10.5688/ajpe7710218

11. Glassick CE. Boyer's expanded definitions of scholarship, the standards for assessing scholarship, and the elusiveness of the scholarship of teaching. Acad Med. 2000;75(9):877-880. doi: 10.1097/00001888200009000-00007

12. TCPS 2: CORE - Tutorial. Canadian Panel of Research Ethics. https://tcps2core.ca/welcome. Accessed July 7, 2021.

13. UBC Office of Research Ethics. Checklist for quality improvement/quality assurance/program evaluation/curriculum development studies requiring ethical review.

https://ethics.research.ubc.ca/sites/ore.ubc.ca/files/doc uments/BREB ChecklistForResearchRequiringEthicsRevi ew.pdf. Accessed July 7, 2021.

14. BREB application guide: A SoTL-specific resource. UBC Institute for the Scholarship of Teaching and Learning. https://isotl.ctlt.ubc.ca/breb-application/. Accessed July 7, 2021.

15. Pope KS, Vasquez MJT. Ethics in Psychotherapy and Counseling: A Practical Guide, 5th Edition. Hoboken, NJ: Wiley; 2016.

16. Hicks RW. Maintaining ethics in quality improvement. AORN J. 2016;103(2):139-141. doi: 10.1016/j.aorn.2015.12.014

17. Regehr G. It's NOT rocket science: rethinking our metaphors for research in health professions education. Med Educ. 2010;44(1):31-39. doi: 10.1111/j.13652923.2009.03418.x 
18. Stockley D, Balkwill LL. Raising awareness of research ethics in SoTL: the role of educational developers. CJSoTL. 2013;4(1): Article 7. doi: 10.5206/cjsotlrcacea.2013.1.7

19. MacLean M, Poole G. An introduction to ethical considerations for novices to research in teaching and learning in Canada. CJSoTL. 2010;1(2): Article 7. doi: 10.5206/cjsotl-rcacea.2013.1.7

20. Poole G. Square one: What is research? In: Kathleen McKinney, ed. The scholarship of teaching and learning in and across the disciplines. Bloomington, IN: Indiana University Press; 2013:135-151.
21. Fedoruk, LM, Mikita, K,. The role of educational developer in supporting research ethics in SoTL. In: Plews RC, Amos ML, eds. Evidence-Based Faculty Development Through the Scholarship of Teaching and Learning (SoTL). Hershey PA : IGI Global Publishers; 2020:221-236. doi: 10.4018/978-1-7998-2212-7.ch012

22. Booth S, Woollacott L. On the constitution of SoTL: its domains and contexts. High Educ. 2018;75(2):527-551. doi: 10.1007/s10734-017-0156-7

[1] The TCPS is a joint policy of Canada's three federal research funding agencies (Canadian Institutes of Health Research, the National Sciences and Engineering Institute of Canada and the Social Sciences and Humanities Research Council) expressing their continuing commitment to the people of Canada to promote the ethical conduct of research involving human subjects. This commitment was first expressed in guidelines published in the 1970s with numerous amendments since then. The most recent policy statement (TCPS 2) was published in 2018.

[2] Abbreviations used in this commentary include: TCPS (Tri-Council Policy Statement), TCPS 2 (Tri-Council Policy Statement, the most recent 2018 version), REB (Research Ethics Board), BREB (Behavioral Research Ethics Board), QA/QI (quality assurance/quality improvement), ORE (Office of Research Ethics, UBC's ethics oversight body), CTLT (Centre for Teaching, Learning and Technology, UBC's enterprise-level teaching and learning center), and RISe (UBC's enterprise-level Research Information System).

[3] The literature search utilized the UBC Library Collections search engine. Search parameters included peer-reviewed publications from January 2009 to September 2019 using the query: (ethics review or research ethics review) and (education or pedagogy) and (higher education or pharmacy or medicine or medical or nursing or dentistry or health professions). Titles and abstracts were triaged for English language, primary literature articles that addressed: 1) challenges/barriers faced in research ethics review of pedagogical studies, and/or; 2) guidance on navigating research ethics review in pedagogical studies. Critical reading of search results yielded a small subset of 12 articles most relevant to the project context. The references cited in these papers were used to expand the literature base.

[4] The guide has been written for pharmacy education researchers. Reference to the PERL stream refers to the Pharmacy Education Research and Leadership research theme, one of four core research areas in our Faculty. PERL members include pharmacy educators actively engaged in educational scholarship and SoTL studies.

[5] Acknowledging educational scholarship as research or QA/QI as defined by the TCPS 2 is important for dissemination purposes. The former requires the BREB certificate approval number obtained as part of the formal ethics review process while the latter can be recognized by including specific language provided by UBC's ORE such as "According to Article 2.5 of the TCPS 2, this project was deemed a quality assurance/quality improvement study and therefore not subject to formal institutional ethics review."

[6] Inferred guidance regarding SoTL in the TCPS $2^{1}$ is included in Article 3.2, paragraph (e) which states that "researchers should separate, to the greatest degree possible, their roles as researcher from their other roles as therapists, caregivers, teachers, advisors, consultants, supervisors, employers or the like" (p. 32). 